\title{
Bacterial Identification and Monitoring Around Two-Piece Dental Implants by Matrix-Assisted Laser Desorption Ionization Time of Flight Mass Spectrometry (MALDI-TOF MS)
}

\author{
Sonali Saha ${ }^{1}$ Ajita Meenawat ${ }^{1}$ Chinmoy Sahu ${ }^{2}$ Vivek Srivastava $^{1}$ Shivam Yadav ${ }^{1}$ Vinod Kumar $^{1}$ \\ ${ }^{1}$ Department of Periodontology and Implantology, Sardar Patel Post \\ Graduate Institute of Dental and Medical Sciences, Lucknow, India \\ 2Department of Microbiology, Sanjay Gandhi Post Graduate \\ Institute of Medical Sciences, Lucknow, Uttar Pradesh, India \\ Address for correspondence Chinmoy Sahu, Department of \\ Microbiology, Sanjay Gandhi Post Graduate Institute of Medical \\ Sciences, Lucknow, Uttar Pradesh 226014, India \\ (e-mail: sahu.chinmoy@gmail.com).
}

J Lab Physicians:2020;12:49-55

\begin{abstract}
Context Early microbiological diagnosis and treatment of periodontal pathogens is important for successful retention of dental implants.

Aims This study aimed to identify and monitor oral bacterial colonization after successful two-piece dental implants.

Settings and Design In this study, 50 two-piece dental implant subjects were included and assessed clinically, radiographically, and microbiologically.

Methods and Material All the parameters were recorded at various stages after prosthesis placement. In each stage, nonadherent (peri-implant sulcular fluid) and adherent (curetted inner threads) samples were collected. Semiquantitative anaerobic culture of the samples were done in Anoxomat system. Bacterial colonies were first identified by routine microbiological methods and then by matrix-assisted laser desorption ionization-time of flight mass spectrometry (MALDI-TOF MS) method.

Statistical Analysis All the results were analyzed by appropriate statistical methods (Chi-square, one factor analysis of variance, etc.).

Results All the bacterial isolates were identified in the MALDI-TOF MS system with no failure. After implant placement for the nonadherent samples, the frequency (\%) of Fusobacterium nucleatum, Prevotella melaninogenica, and Propionibacterium acnes decreased whereas frequency (\%) of Escherichia coli, Staphylococcus epidermidis, and Streptococcus mitis increased. For adherent samples, the frequency (\%) of E. coli, Enterococcus faecalis, Porphyromonas gingivalis, P. melaninogenica, and Veillonella par-

Keywords

- dental implant

- bacteria

- MALDI-TOF MS

- monitoring

- culture vula decreased, whereas frequency (\%) of S. mitis and Streptococcus mutans increased. The postimplant mean nonadherent and adherent bacterial load increased with time but not significantly over the periods ( $p=0.302$ and 0.123 , respectively).

Conclusion Combination of basic (semiquantitative culture method) and advanced microbiological method (MALDI-TOF MS) can be useful for accurate detection and monitoring of potential pathogens around two-piece dental implants.
\end{abstract}




\section{Introduction}

Most of dental implants are two-piece systems comprising an endosteal implant body and suprastructure-carrying abutment. The two components (i.e., implant body and abutment) are fitted at the implant-abutment interface. ${ }^{1}$ Soft tissue relationship to implants is one of the most challenging areas in implant dentistry and is influenced by various contributing factors like surgical protocol, implant and abutment design, and loading considerations.

There is difference between tooth and implant biologic attachments which make dental implants more susceptible to inflammation. ${ }^{2,3}$ The process and extent to which microorganism colonize intraimplant cavities and form biofilm on intraimplant surfaces after implant placement remain unclear. ${ }^{4,5}$ The periodontium should be checked and its healthy status must be maintained for early detection of pathogens and implant maintenance. ${ }^{6}$

The microbiologic investigations are usually done for implant patients who are susceptible to periodontal disease for early detection of periodontal pathogens. However, limited literature is available on the microbial colonization of the peri-implant sulcus area without clinical periodontal disease. It is important to detect the oral bacterial flora to their species level. Various techniques that have been used for microbial detection are routine bacterial culture, automated culture systems, DNA-DNA hybridization, and polymerase chain reaction.

A new diagnostic technique, matrix-assisted laser desorption ionization-time of flight mass spectrometry (MALDI-TOF MS) expanded the field and allowed for the analysis of biological molecules. ${ }^{7}$ In an increasing number of settings, MALDI-TOF MS has replaced traditional identification methods, including microscopy and in determination of phenotypic characteristics, which typically requires multiple steps. ${ }^{89}$ In comparison to conventional methods, this technology is much less labor intensive and can provide accurate and reliable results in minutes from single isolated colony.

At present, only few data are available regarding the oral microorganism colonizing the internal surfaces of two-piece dental implants. Thus, the present study has been undertaken to evaluate periodontal pathogens around two-stage dental implants during various stages of implant rehabilitation using MALDI-TOF MS technique.

\section{Subjects and Methods}

The present study was conducted in the Department of Periodontology and Implantology, in collaboration with Department of Microbiology. Patients were explained about the study procedures and a written informed consent was taken from the patients who were willing to participate in the study and ethical clearance was obtained from Institutional Ethical Committee Review Board.

Subjects were selected from the outpatient pool of the Department of Periodontology \& Implantology. Total 50 sites where implants were placed and had undergone second stage implant surgery 1 week before were included in the study.

The patients fulfilling the following criteria were included in the study: patients aged between 18 and 60 years, patients motivated to maintain oral hygiene and understood maintenance protocols, and medically fit patients.

The following patients were excluded from the study: history of antibiotics use during and 1 month before prosthetic loading, implants with evidence of peri-implantitis assessed clinically and radiographically, immunocompromised patients, pregnant or lactating females, current smokers and tobacco chewers, acute or chronic systemic disorders (e.g., uncontrolled diabetes, autoimmune deficiency).

Patients included in the study were informed about the study and written consent was taken. Clinical examination was done on all patients, which consisted of a thorough medical and dental history, general and oral health status, and assessment of implant site.

A total of 50 implants were included in the study and assessed clinically, radiographically, and microbiologically.

Clinical parameters that were assessed for two-stage dental implants are: modified plaque index (MPI), modified sulcus bleeding index (MSBI), and peri-implant probing depth (PPD). ${ }^{10}$

All the above parameters were recorded at various stages namely-2 days after prosthesis placement (Appointment B1), 7 days after prosthesis placement (Appointment B2), and 1 month after prosthesis placement (Appointment B3).

Sampling from implant sites were performed at various time frames (B1, B2, and B3). Before sampling, the implant site was isolated with sterile gauze pads to prevent contamination by saliva. For nonadherent microbial samples, paper points were passively inserted in the peri-implant sulcus. The fixture was washed using 1-mL syringe and was filled with $100-\mu \mathrm{L}$ sterile $\mathrm{NaCl}$ solution. For adherent plaque, plastic curettes were inserted into the deepest part of the implant and the inner threads were brushed twice in counterclockwise direction. Thereafter, the samples were placed into sterile tubes filled with 1.95-mL prereduced culture medium (Robertson's cooked meat medium) such that only minimal air volume remained. Within half an hour the samples were sent to the Department of Microbiology for microbiological assessment of the samples. Patients were advised to follow implant maintenance criteria. ${ }^{11}$

The nonadherent microbial samples were inoculated in serial dilutions onto sheep blood agar and Wilkins-Chalgren agar. The thioglycolate broth tubes, containing the microbrushes, were vortexed and then were stirred by rotation for 20 minutes. Aliquots of the microbial suspensions were plated onto sheep blood agar and Wilkins-Chalgren agar.

Blood agar plates were incubated in air enriched with $10 \%$ $\mathrm{CO}_{2}$, whereas Wilkins-Chalgren agar plates were placed in anaerobic jars for up to 7 days at $37^{\circ} \mathrm{C}$. Anaerobic condition was created by Anoxomat System (Advanced Instruments).

To set up a jar for anaerobic conditions, the jar was attached to the Anoxomat by a lead which formed a gas tight fit by means of a snap-shut adaptor on the lid. The jar was then evacuated by 
the selected method after which an audible signal was given to indicate completion of the cycle as given by Brazier et al. ${ }^{12}$

Representative colonies were isolated according to growth morphology, pigmentation, and hemolysis. All isolates were subjected to various microbiological methods for identification. Organisms were quantified in colony-forming units (CFU) per milliliter for each sample tested according to Hereker et al. ${ }^{13}$

The bacterial isolates were first stained by Gram staining to differentiate between gram-positive bacteria and gram-negative bacteria. The identities were further confirmed by biochemical tests as given by Dowell and Hawkins. ${ }^{14}$

The MALDI-TOF assay was performed in VITEK-MS (bioMérieux SA) system. Pure cultures of bacterial isolates were processed. First, the provided disposable plate containing 48 spots was taken. With a wooden applicator, bacterial spots were prepared on the plate. The spot of control bacterial strain (E. coli ATCC 8739) was prepared on the control spot. Then the spots of test isolates were made. After that, $1 \mu \mathrm{L}$ of provided matrix ( $\alpha$-cyanohydroxy 4 -cinnamic acid) was put on each spot by a micropipette. ${ }^{15}$

After all the spots of bacterial isolates were made, the plate barcodes were scanned by prep-station scanner and the plates were put in the VITEK-MS. The machine gave the results in 15 to 20 minutes.

\section{Statistical Analysis}

Data were summarized as mean \pm SE (standard error of the mean). Groups were compared by one factor analysis of variance (ANOVA) and the significance of mean difference between (inter) the groups was done by Tukey's HSD (honestly significant difference) post hoc test after ascertaining normality by Shapiro-Wilk's test and homogeneity of variance between groups by Levene's test. Categorical (discrete) groups were summarized in number $(n)$ and percentage (\%) and compared by chi-square $\left(\chi^{2}\right)$ test. A two-tailed $(\alpha=2) p<0.05$ was considered statistically significant. Analysis was performed on SPSS version 17.0 (IBM Corp.).

\section{Results}

The periodontal parameters (MPI, MSBI) did not increase significantly over the follow-up period except PPD (-Table 1).
It indicated that the peri-implant tissue health can be maintained if the patients are aware about the benefits of good oral hygiene after implant therapy.

All the bacterial isolates were identified in the MALITOF system with no failure. After implant placement, the frequency (\%) of Fusobacterium nucleatum, Prevotella melaninogenica, Propionibacterium, Staphylococcus aureus, Streptococcus salivarius, Streptomyces griseus, and Veillonella parvula decreased, whereas frequency (\%) of $E$. coli, Staphylococcus epidermidis, Streptococcus mitis, and Streptococcus mutans increased. In contrast, the frequency (\%) of both Prevotella intermedia and Veillonella atypica

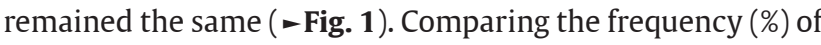
nonadherent plaque bacteria of three periods, $\chi^{2}$ test showed similar frequency of nonadherent plaque bacteria among the periods ( $\chi^{2}=16.17, p=0.963$ ), i.e., frequency (\%) did not differ significantly.Further, the nonadherent plaque bacterial load at appointments B1, B2, and B3 ranged from 2 to $400 \times 10^{4}$ $\mathrm{CFU} / \mathrm{mL}, 1$ to $500 \times 10^{4} \mathrm{CFU} / \mathrm{mL}$, and 2 to $450 \times 10^{4} \mathrm{CFU} / \mathrm{mL}$, respectively with mean $( \pm \mathrm{SE}) 53.26 \pm 12.61 \times 10^{4} \mathrm{CFU} / \mathrm{mL}$, $65.43 \pm 14.01 \times 10^{4} \mathrm{CFU} / \mathrm{mL}$, and $84.25 \pm 15.84 \times 10^{4} \mathrm{CFU} /$ $\mathrm{mL}$, respectively, and median of 20,30 , and $27 \times 10^{4} \mathrm{CFU} / \mathrm{mL}$, respectively. The post treatments mean nonadherent plaque bacterial load increased with time but not significantly over the periods $(F=1.21, p=0.302)(-$ Fig. 2 ).

Also, in the adherent samples, MALDI-TOF detected all bacterial isolates. After implant placement, the frequency (\%) of E. coli, Enterococcus faecalis, Porphyromonas gingivalis, $P$. melaninogenica, and $V$. parvula decreased, whereas $S$. mitis and $S$. mutans increased. In contrast, the frequency (\%) of Neisseria subflava, P. intermedia, Propionibacterium acnes, S. aureus, and S. salivarius remained the same (-Fig. 3). Comparing the frequency of adherent plaque bacteria of three periods, $\chi^{2}$ test showed similar frequency of adherent plaque bacteria among the periods $\left(\chi^{2}=12.31, p=0.998\right)$, that is, the frequency did not differ significantly.

Further, the adherent plaque bacterial load at B1, B2, and B3 ranged from 2 to $500 \times 10^{4} \mathrm{CFU} / \mathrm{mL}, 2$ to $240 \times 10^{4} \mathrm{CFU} /$ $\mathrm{mL}$, and 2 to $650 \times 10^{4} \mathrm{CFU} / \mathrm{mL}$, respectively with mean $( \pm \mathrm{SE})$ $36.60 \pm 12.38 \times 10^{4} \mathrm{CFU} / \mathrm{mL}, 49.72 \pm 10.37 \times 10^{4} \mathrm{CFU} / \mathrm{mL}$, and $74.72 \pm 16.36 \times 10^{4} \mathrm{CFU} / \mathrm{mL}$, respectively and median $10 \times 10^{4} \mathrm{CFU} / \mathrm{mL}, 20$, and $25 \times 10^{4} \mathrm{CFU} / \mathrm{mL}$, respectively. The post treatments mean adherent plaque bacterial load increased with time but not significantly $(F=2.13, p=0.123)$ (-Fig. 4).

Table 1 The different periodontal parameters (MPI, MSBI, and PPD) in the three appointments

\begin{tabular}{|c|c|c|c|c|c|c|c|}
\hline Appointment & Periods & $\begin{array}{l}\text { MPI } \\
\text { Mean } \pm S E \\
n=50\end{array}$ & $p$-Value & $\begin{array}{l}\text { MSBI } \\
\text { Mean } \pm \text { SE, } \\
n=50\end{array}$ & $p$-Value & $\begin{array}{l}\text { PPD } \\
\text { Mean } \pm S E \text {, } \\
n=50\end{array}$ & $p$-Value \\
\hline B1 & $\begin{array}{l}\text { Two days after gingival } \\
\text { former placement }\end{array}$ & $0.38 \pm 0.07$ & \multirow[t]{3}{*}{0.378} & $0.24 \pm 0.06$ & \multirow[t]{3}{*}{0.213} & $2.36 \pm 0.07$ & \multirow[t]{3}{*}{$<0.001$} \\
\hline B2 & $\begin{array}{l}\text { One week after prosthesis } \\
\text { placement }\end{array}$ & $0.38 \pm 0.07$ & & $0.36 \pm 0.07$ & & $2.90 \pm 0.06$ & \\
\hline B3 & $\begin{array}{l}\text { One month after prosthesis } \\
\text { placement }\end{array}$ & $0.50 \pm 0.07$ & & $0.40 \pm 0.07$ & & $3.10 \pm 0.05$ & \\
\hline
\end{tabular}

Abbreviations: MPI, modified plaque index; MSBI, modified sulcus bleeding index; PPD, peri-implant probing depth; SE, standard error. 


\section{Non adherent plaque bacteria}

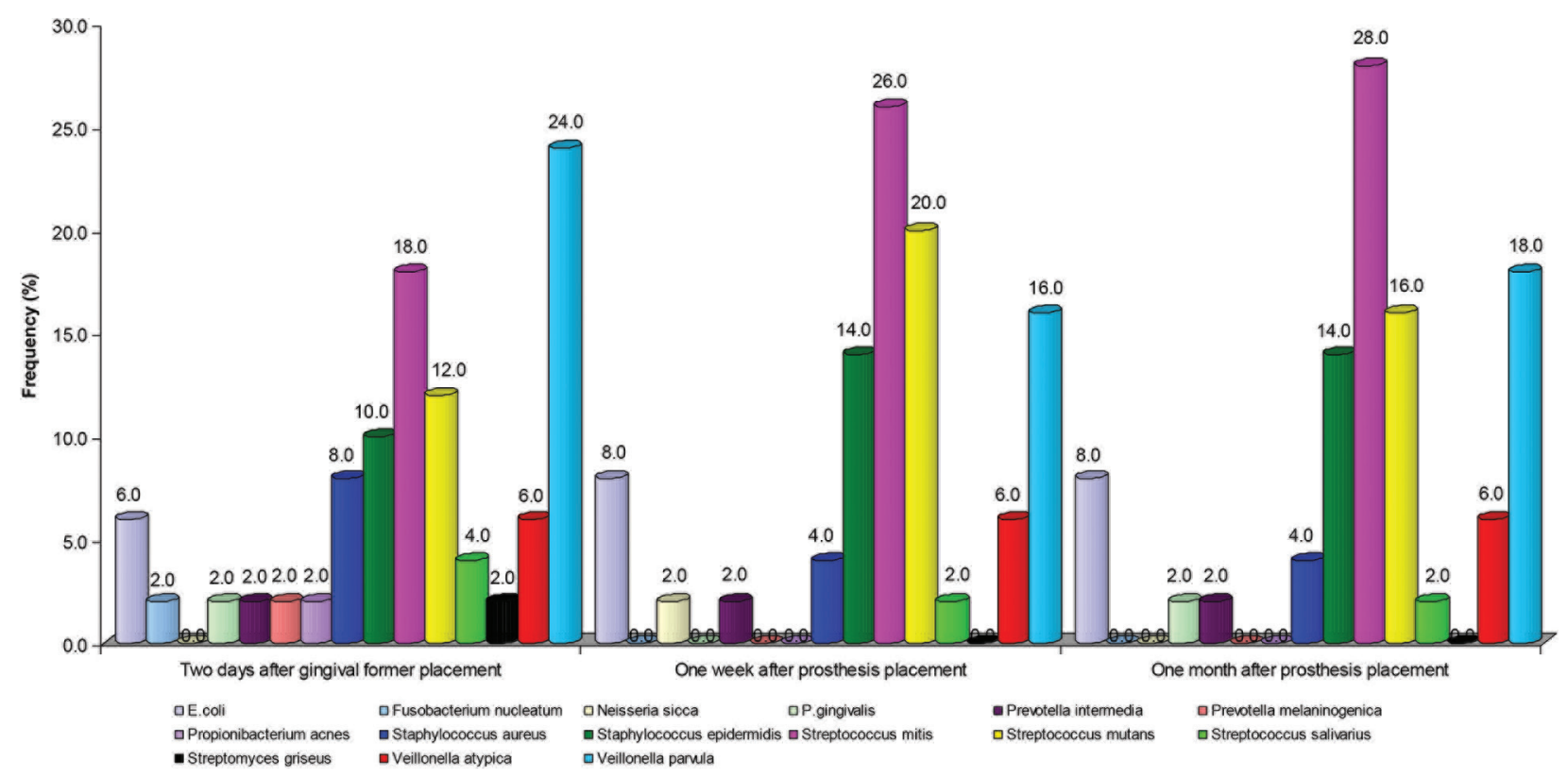

Fig. 1 Nonadherent plaque bacteria.

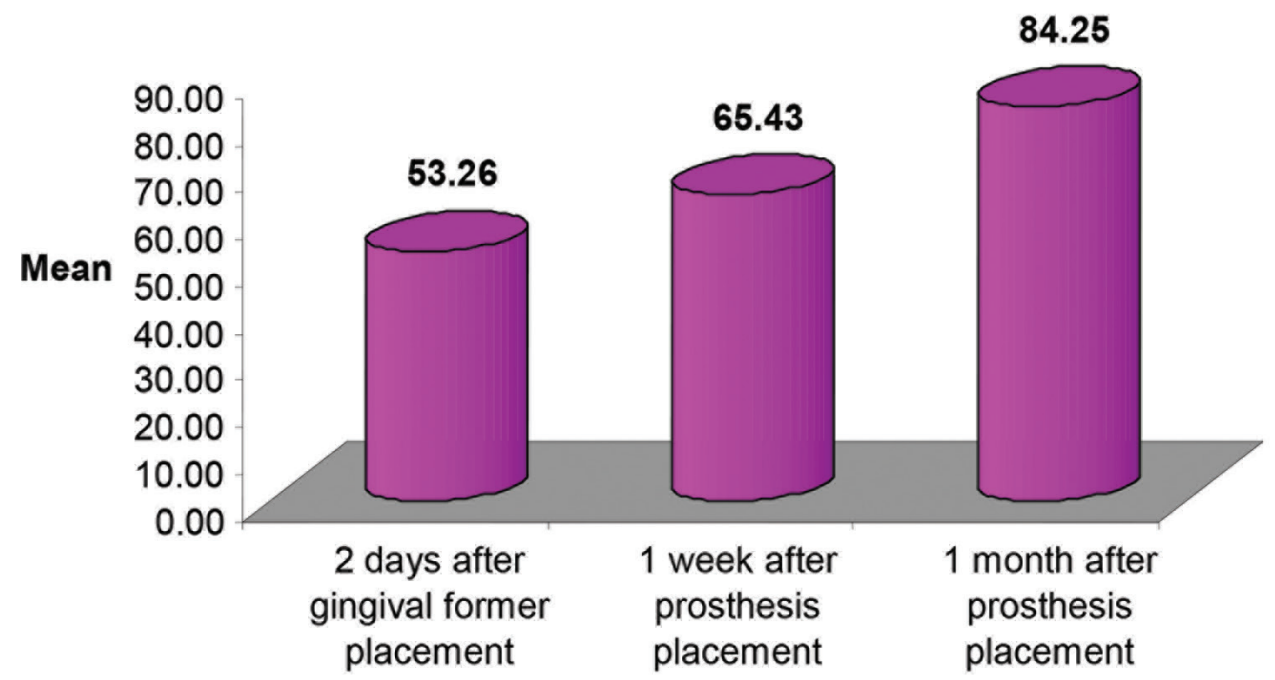

Fig. 2 Nonadherent plaque bacterial load.

\section{Discussion}

This descriptive study surveyed bacterial detection and load in adherent and nonadherent plaque samples around two-stage dental implants. The periodontal parameters such as MPI, MSBI, and PPD were followed up in the different appointment. The MPI and MSBI did not change significantly which indicate the subjects of the present study have followed the implant maintenance protocol properly and maintained good oral hygiene after implant placement.

In the study, samples collected at different time intervals demonstrated a consistency in the occurrence of certain pathogens during the entire experimental period.
Nonadherent flora (denotes transient flora) and adherent flora (denotes established bacteria) around two-stage dental implants at various stages were evaluated. The use of MALDITOF significantly increased the accuracy of the identity of bacteria and the rapidity of identification as compared with traditional methods of bacterial identification.

In both nonadherent and adherent samples, loads of $E$. coli, S. epidermidis, S. mitis, and S. mutans were increased. These bacteria have the propensity to attach to implant surfaces and produce biofilms. ${ }^{16}$ Also they form capsules around their cell wall, the property further recruits and attaches more bacteria of their kind. After prosthetic loading the surface area increases around implant which leads to more 


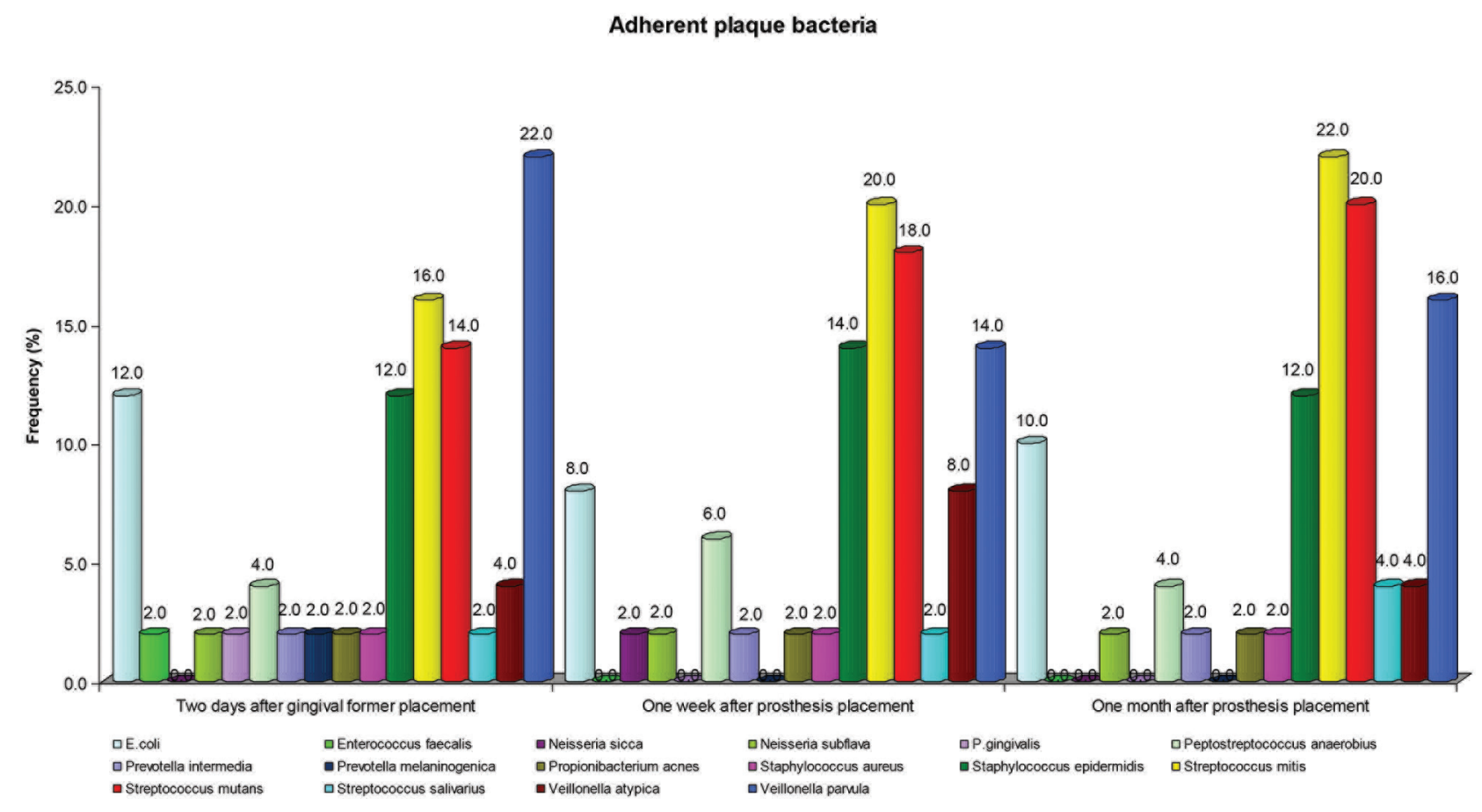

Fig. 3 Adherent plaque bacteria.

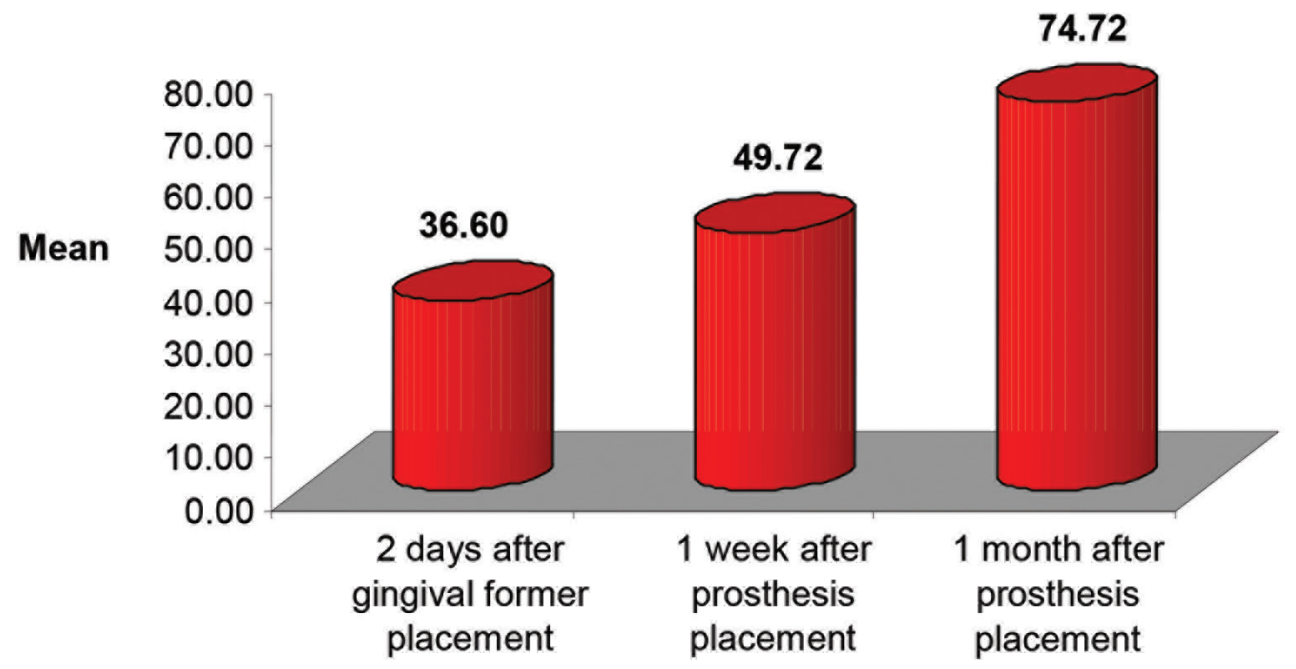

Fig. 4 Adherent plaque bacterial load.

accumulation of bacteria and therefore leads to increase in bacterial load. So, their numbers gradually increase as detected in the present study. On the other hand, other bacteria have more planktonic or mobile forms which are gradually washed out if good hygiene protocol is maintained. So, their count remains stable or decreased over a time period, which is necessary for long-term implant survival.

Oral Streptococci are gram-positive bacteria that comprise of various species like S. mutans, S. mitis, and Streptococcus gordonii. This group was the second most common abundant group in the study with respect to both nonadherent and adherent bacteria (16 and 30\%, respectively). Hereker et al reported $85 \%$ prevalence of streptococci in their study. ${ }^{13}$
Also these findings were reinforced by Asadzadeh et al and Mencio et al. ${ }^{17,18}$ This bacteria is related to the health of periodontium as well as implants, so the results depict that subjects have maintained proper oral hygiene instruction after implant placement.

Two major pathogens, i.e., A. actinomycetemcomitans (0\%) and $P$. gingivalis (2\%) were extremely low at B1, B2, and B3 in both nonadherent and adherent samples in this study. Similar findings were reported by van Winkelhoff et al. ${ }^{19}$ Hereker et al also reported very less prevalence (1\%) of $P$. gingivalis in their study. ${ }^{13}$

The $P$. intermedia group bacteria are black-pigmented gram-negative anaerobic rods that are commonly found in 
oral biofilm. P. intermedia is associated with periodontal disease and Prevotella nigrescens with a healthy periodontium. ${ }^{20}$ Routine biochemical tests and gas-liquid chromatography are incapable of separating them. ${ }^{21}$ These techniques are also time-consuming and have low levels of reproducibility. MALDI-TOF MS is good and rapid method to identify P. intermedia and $P$. nigrescens. ${ }^{22}$ In the study, low prevalence (2\%) of $P$. intermedia was also detected by MALDI-TOF MS in both nonadherent and adherent samples, which indicated better prognosis of implants in the subjects. No P. nigricans was detected. Hereker et al also reported extremely low prevalence of $P$. intermedia in their study using conventional identification methods. ${ }^{13}$

Veillonella spp. (V. parvula and V. atypica) is a gram-negative oral bacteria. In the study, high prevalence of this bacteria as 30 and $26 \%$ in nonadherent and adherent samples respectively was found. High prevalence of this bacteria (43.8\%) was also described by Buchmann et al. ${ }^{23}$ In a microbiological study of dental implants, Lee et al reported $V$. parvula to be the most abundant followed by Streptococcus sanguis. ${ }^{24}$ However, the pathogenicity of Veillonella is low as compared with other established periodontal bacteria. ${ }^{25}$

Further, mean nonadherent plaque bacterial load increased with time. However, the mean nonadherent plaque bacterial load between the periods, did not differ significantly $(p>0.05)$. Similarly, the mean adherent plaque bacterial load between the periods did not differ significantly $(p>0.05)$. This similarity in maximum CFU/mL between the periods (B2 and B3) indicating the establishment of equilibrium after prosthesis placement; this denotes that subjects were following good implant maintenance protocol.

Hereker et al reported similar bacterial load in nonadherent and adherent plaque samples around dental implants. ${ }^{13}$ The loads of various bacteria changed but not significantly during the follow-up period of 1 month, as found in the study. Harder et al reported mean bacterial load to be $41 \times 10^{4}$ $\mathrm{CFU} / \mathrm{mL}$ in nonadherent samples only. ${ }^{1}$ However, they did not study the change in bacterial load over a time period.

In 1992, Socransky and Haffajec modified the postulates of Koch establishing criteria that identify periodontal pathogens. ${ }^{26}$ While the "purple" (with fundamentally aerobic flora lacking mobility), "yellow" and "green" complexes are not associated with disease, the "orange" (F. nucleatum, P. intermedia, Peptostreptococcus micros) and "red" complexes (P. gingivalis, Tannerella forsythia, Treponema denticola) are disease-related. The presence of a pathogenic microflora in an initial phase can be associated with a higher risk of peri-implantitis. $^{27,28}$ The present study detected the bacteria of orange and red complexes, but the prevalence was very low (2\%). The subjects in the present study with red and orange complex pathogens around dental implants are prone to develop peri-implant diseases, so they have to follow a strict implant maintenance protocol and regular follow-ups to prevent early implant loss.

There is a need to identify the patients who have prevalence to harbor the pathogenic microorganisms. Microbiological tests may be a valuable tool for differential diagnosis of problems occurring with implant osseointegration and peri-implantitis. As the organisms continue to inhabit the oral cavity, these patients need better monitoring regarding oral hygiene. The proper and rapid identification of bacteria is very important for this purpose.

The early and accurate detection of potential pathogens around the dental implants is essential so that strict hygienic protocol can be maintained to prevent peri-implantitis and implant failure. Conventional identification methods take longer time and all anaerobic species cannot be determined by these methods. These methods are also costly and labor intensive. MALDI-TOF is helpful in this situation because this method is very rapid (takes 15 minutes) and very accurate because it utilizes the proteomics of bacteria which are not altered. The average cost per test is also very less compared with conventional methods. For this purpose, this technique is replacing the conventional methods in bacterial identification. In this study also, MALDI-TOF led to early and accurate identification of microflora around dental implants so that proper infection control measures are advised to prevent pathogenic bacterial colonization and early implant loss.

\section{Funding \\ None.}

\section{Conflict of Interest}

The authors declare no conflict of interest.

\section{References}

1 Harder S, Podschun R, Grancicova L, Mehl C, Kern M. Analysis of the intraimplant microflora of two-piece dental implants. Clin Oral Investig 2013;17(4):1135-1142

2 Sanctis MD, Baldini N, Vignoletti F. Biological width around implants. Histological evidence: a review on animal studies. J de Parodontologie Orale 2010;29:4-17

3 Inoue T, Peri-implant Tissue. In: Izumi Y, Kodama T, Matsui T, eds. Approach to Periimplantitis. Tokyo, Japan: Agasueshoten Co., Ltd; 2007 6-11

4 Cosyn J, Van Aelst L, Collaert B, Persson GR, De Bruyn H. The peri-implant sulcus compared with internal implant and suprastructure components: a microbiological analysis. Clin Implant Dent Relat Res 2011;13(4):286-295

5 D'Ercole S, Tetè S, Catamo G, et al. Microbiological and biochemical effectiveness of an antiseptic gel on the bacterial contamination of the inner space of dental implants: a 3-month human longitudinal study. Int J Immunopathol Pharmacol 2009;22(4):1019-1026

6 Silverstein LH, Kurtzman D, Garnick JJ, Schuster GS, Steflik DE, Moskowitz ME. The microbiota of the peri-implant region in health and disease. Implant Dent 1994;3(3):170-174

7 Karas M, Hillenkamp F. Laser desorption ionization of proteins with molecular masses exceeding 10,000 Daltons. Anal Chem 1988;60(20):2299-2301

8 Buchan BW, Ledeboer NA. Advances in identification of clinical yeast isolates by use of matrix-assisted laser desorption ionization-time of flight mass spectrometry. J Clin Microbiol 2013;51(5):1359-1366

9 De Carolis E, Vella A, Vaccaro L, et al. Application of MALDI-TOF mass spectrometry in clinical diagnostic microbiology. J Infect Dev Ctries 2014;8(9):1081-1088

10 Mombelli A, van Oosten MA, Schurch E Jr, Land NP. The microbiota associated with successful or failing osseointegrated titanium implants. Oral Microbiol Immunol 1987;2(4): 145-151 
11 Humphrey S. Implant maintenance. Dent Clin North Am 2006;50(3):463-478, viii

12 Brazier JS, Smith SA. Evaluation of the Anoxomat: a new technique for anaerobic and microaerophilic clinical bacteriology. J Clin Pathol 1989;42(6):640-644

13 Herekar M, Sethi M, Prithviraj DR, Bhat K, Fernandes A, Patil V. A clinical study evaluating changes in the microbial flora around dental implants during various stages of implant restoration. Implant Dent 2015;24(5):527-532

14 Dowell VR, Hawkins TM, Laboratory Methods in Anaerobic Bacteriology. CDC Laboratory Manual. Atlanta: Centers for Disease Control; 1987

15 Guo L, Ye L, Zhao Q, Ma Y, Yang J, Luo Y. Comparative study of MALDI-TOF MS and VITEK 2 in bacteria identification. J Thorac Dis 2014;6(5):534-538

16 Matsumoto-Nakano M. Role of Streptococcus mutans surface proteins for biofilm formation. Jpn Dent Sci Rev 2018;54(1):22-29

17 Asadzadeh N, Naderynasab M, Fard FG, Rohi A, Haghi HR. The microbiology of the peri-implant sulcus following successful implantation of oral prosthetic treatments. Indian J Dent Res 2012;23(6):753-757

18 Mencio F, De Angelis F, Papi P, Rosella D, Pompa G, Di Carlo S. A randomized clinical trial about presence of pathogenic microflora and risk of peri-implantitis: comparison of two different types of implant-abutment connections. Eur Rev Med Pharmacol Sci 2017;21(7):1443-1451

19 van Winkelhoff AJ, Loos BG, van der Reijden WA, van der Velden U. Porphyromonas gingivalis,Bacteroides forsythusand other putative periodontal pathogens in subjects with and without periodontal destruction. J Clin Periodontol 2002;29(11):1023-1028
20 Hansen T, Kunkel M, Kirkpatrick CJ, Weber A. Actinomyces in infected osteoradionecrosis-underestimated? Hum Pathol 2006;37(1):61-67

21 De Ceulaer J, Tacconelli E, Vandecasteele SJ. Actinomyces osteomyelitis in bisphosphonate-related osteonecrosis of the jaw (BRONJ): the missing link? Eur J Clin Microbiol Infect Dis 2014;33(11):1873-1880

22 Stîngu CS, Rodloff AC, Jentsch H, Schaumann R, Eschrich K. Rapid identification of oral anaerobic bacteria cultivated from subgingival biofilm by MALDI-TOF-MS. Oral Microbiol Immunol 2008;23(5):372-376

23 Buchmann R, Khoury F, Pingel D, Lange DE. The microflora recovered from the outer-surfaces of the Frialit-2 implanto-prosthetic connector. Clin Oral Implants Res 2003;14(1): $28-34$

24 Lee KH, Maiden MF, Tanner AC, Weber HP. Microbiota of successful osseointegrated dental implants. J Periodontol 1999;70(2):131-138

25 Socransky SS, Haffajee AD. Effect of therapy in periodontal infections. J Periodontol 1993;64:754-759

26 Socransky SS, Haffajee AD. The bacterial etiology of destructive periodontal disease: current concepts. J Periodontol 1992;6:322-331

27 Scapoli L, Girardi A, Palmieri A, et al. Quantitative analysis of periodontal pathogens in periodontitis and gingivitis. J Biol Regul Homeost Agents 2015;29(3, suppl 1) :101-110

28 Quaranta A, Andreana S, Pompa G, Procaccini M. Active implant peri-apical lesion: a case report treated via guided bone regeneration with a 5-year clinical and radiographic follow-up. J Oral Implantol 2014;40(3):313-319 